\title{
Status and performance of the CALIOP lidar
}

\author{
David M. Winker*a ${ }^{\mathrm{a}}$, William Hunt ${ }^{\mathrm{b}}$, and Chris Hostetler ${ }^{\mathrm{a}}$ \\ ${ }^{a}$ NASA Langley Research Center, MS/435, Hampton, Virginia 23681, USA \\ ${ }^{*}$ Wyle Laboratories, Hampton, Virginia, USA
}

\begin{abstract}
The Cloud-Aerosol LIdar with Orthogonal Polarization (CALIOP) is the primary instrument on the CALIPSO satellite, which is scheduled to launch in 2005. CALIOP will provide profiles of total backscatter at two wavelengths, from which aerosol and cloud profiles will be derived. The instrument also measures the linear depolarization of the backscattered return, allowing discrimination of cloud phase and the identification of the presence of non-spherical aerosols. CALIOP is complete and has been tested in a ground-based configuration. This paper provides information on basic characteristics and performance of CALIOP.
\end{abstract}

\section{INTRODUCTION}

The Cloud-Aerosol Lidar and Infrared Pathfinder Satellite Observations (CALIPSO) mission is being developed by NASA Langley Research Center in collaboration with the French Space Agency CNES for launch in $2005^{1}$. CALIPSO will fly at an altitude of $705 \mathrm{~km}$ and an inclination of $98^{\circ}$ as part of the so-called "A-train". The primary objective of the CALIPSO 3-year mission is to provide the observations necessary to improve our understanding of the effects of clouds and aerosols on the climate system. The CALIPSO payload includes three instruments: (1) the Cloud-Aerosol LIdar with Orthogonal Polarization (CALIOP, pronounced the same as "calliope") to provide vertical profiles of aerosol and cloud backscatter and depolarization; (2) an Imaging Infrared Radiometer (IIR) with three channels in the infrared window region optimized for retrievals of cirrus particle size; and (3) the Wide Field Camera (WFC), a moderate spatial resolution imager with one visible channel which provides meteorological context and a means to accurately register CALIPSO observations to those from MODIS on the Aqua satellite. The payload also includes several support systems: a Payload Controller (PLC) and an X-band transmitter subsystem. The payload controller performs control and data handling functions for all three instruments as well as for the X-band transmitter, which is used to downlink instrument science and housekeeping data. The CALIPSO payload was built by the Ball Aerospace and Technology Corporation (BATC) in Boulder, Colorado, except for the IIR which was provided by CNES. The following sections describe the CALIOP instrument, its on-orbit calibration scheme, and performance.

\section{INSTRUMENT DESCRIPTION}

CALIOP consists of a laser transmitter subsystem and a receiver subsystem. Figure 1 shows an exploded view of the "lidar core" contained inside the payload housing. The instrument is built around a T-shaped optical bench which assures stability of the transmitter-to-receiver alinement. The bench is fabricated of a carbon graphite composite material which provides a mechanically and thermally stable structure. The lidar receiver telescope is attached to one side of the bench with the receiver optics and detector assemblies on the other side. The laser transmitter assembly is attached to the top of the " $T$ " by a precision linear drive mechanism and gimbal assembly, allowing precise and accurate pointing adjustments.

\subsection{Transmitter subsystem}

The laser transmitter subsystem includes two identical, redundant laser transmitters, each with a beam expander, and a beam steering system that ensures alinement between the transmitter and receiver. The Nd:YAG lasers produce simultaneous pulses at $1064 \mathrm{~nm}$ and $532 \mathrm{~nm}$ at a pulse repetition rate of $20.16 \mathrm{~Hz}$. The lasers are Q-switched to provide a pulse length of about $20 \mathrm{nsec}$. Each laser generates $220 \mathrm{~mJ}$ of 1064 energy, which is frequency-doubled to produce 110 $\mathrm{mJ}$ of energy at each of the two wavelengths. The two lasers are diode-pumped to maximize their use of the available spacecraft power. Either laser can be selected for operation, but they cannot both be operated simultaneously. Each laser is housed in its own sealed canister filled with dry air at slightly more than standard atmospheric pressure. The output pulse energy at each wavelength is measured using energy monitors located within each canister. Beam expanders reduce

* david.m.winker@nasa.gov; phone 1757 864-6747 
the angular divergence of the transmitted laser beam to produce a beam diameter of 70 meters at the Earth's surface. The lasers are passively cooled using a dedicated thermal radiator panel, avoiding the use of pumps and coolant loops. Transmitter specifications are summarized in Table I.

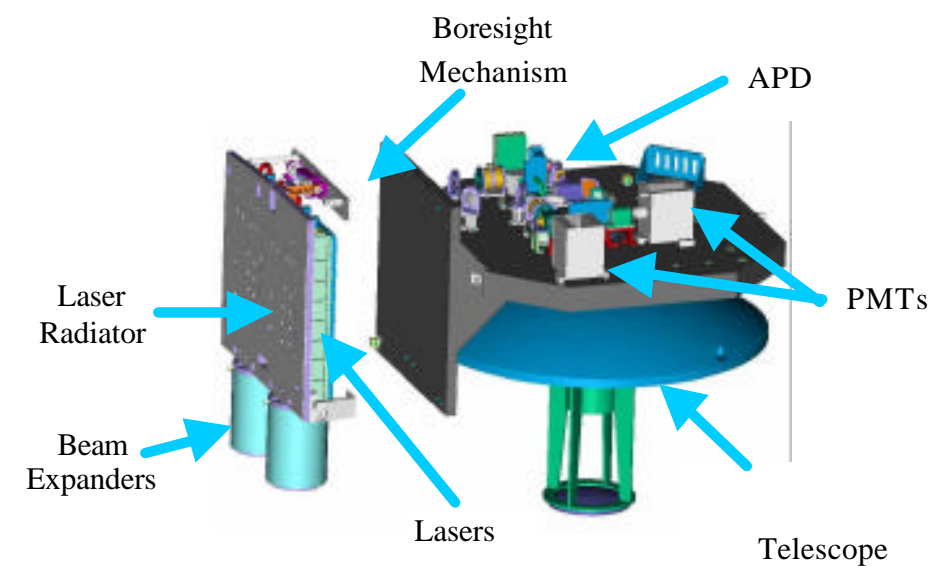

Figure 1. CALIOP transmitter and receiver subsystems.

The lasers were built by Fibertek, Inc. of Herndon, VA, in collaboration with members of the CALIPSO engineering team at Ball and LaRC. Requirements on lifetime and reliability and restrictions on mass and power were major drivers of the design. A ruggedized multi-mode power oscillator design developed by Fibertek for military applications was adopted. A single Nd:YAG slab is pumped by 192 diode bars. A crossed-Porro prism resonator is used to minimize alinement sensitivity, and polarization outcoupling provides a highly polarized output beam. The laser design is capable of producing $400 \mathrm{~mJ}$ per pulse. For CALIPSO, the energy requirement was reduced to $220 \mathrm{~mJ}$ to increase lifetime and reliability. This allowed significant derating of the diode pump current and provided large margins with respect to optical damage thresholds.

Table I. CALIOP transmitter characteristics

\begin{tabular}{|ll|}
\hline Laser & Diode-pumped Nd:YAG \\
Pulse Energy & $110 \mathrm{~mJ}: 532 \mathrm{~nm}$ \\
Rep Rate & $110 \mathrm{~mJ}: 1064 \mathrm{~nm}$ \\
Pulse Length & $20.16 \mathrm{~Hz}$ \\
Linewidth & $20 \mathrm{nsec}$ \\
Polarization Purity & $30 \mathrm{pm}$ \\
Beam Divergence & $>1000: 1(532 \mathrm{~nm})$ \\
& $100 \mu \mathrm{rad}($ after beam \\
Boresight Range & \pm 1 degree, $1.6 \mu \mathrm{rad}$ steps \\
Laser Environment & 18 psia, dry air \\
\hline
\end{tabular}

Effective contamination control is another critical requirement to obtain the necessary lifetime. A space-qualifiable prototype flight laser (the Risk Reduction Laser, or RRL) was built and tested to reduce risk in the development of the flight lasers. The RRL test program was primarily intended to validate the flight laser design and contamination control procedures. Therefore, a comprehensive contamination control plan was developed and applied to the fabrication of the RRL, and later to the flight lasers. An extended lifetest was performed with the RRL, beginning in late 1998. Operating at a pulse rate of $27 \mathrm{~Hz}$, the RRL fired over 2 billion shots during the test, equivalent to the number of shots required for the 
3-year CALIPSO mission, with a measured pulse energy degradation of $6 \%$. This level of degradation was consistent with the results of prior laser diode array lifetests.

\subsection{Receiver subsystem}

Shown schematically in Fig. 2, the receiver sub-system consists of the telescope, relay optics, detectors, preamps, and line drivers, all mounted on a stable optical bench. The completed payload is shown in Fig. 3. Signal processing and control electronics are contained in boxes mounted on the payload housing. The receiver telescope is an all-beryllium 1-meter diameter design similar to the telescope built for the GLAS instrument on the ICESat satellite. The telescope primary mirror, secondary mirror, metering structure, and inner baffle are all made of beryllium, for lightness and to minimize the effect of thermal gradients. A carbon composite light shade prevents direct solar illumination of the mirrors. The telescope is thermally isolated from the optical bench. A field stop at the focus of the telescope defines the receiver field of view of $130 \mathrm{mrad}$ (full angle) and also rejects stray light. A movable shutter placed downstream from the focus blocks light to allow measurements of detector dark current. The shutter mechanism also allows a depolarizer to be moved into the $532 \mathrm{~nm}$ beam for depolarization calibration. A polarization beamsplitter is used to separate the $532 \mathrm{~nm}$ parallel and perpendicular returns. A narrowband etalon is used in combination with a dielectric interference filter in the 532-nm channel to reduce the solar background illumination, while an interference filter alone provides sufficient solar rejection for the $1064 \mathrm{~nm}$ channel. Photomultiplier tubes (PMTs) are used for the $532 \mathrm{~nm}$ detectors as they provide large linear dynamic range, very low dark noise, and reasonable quantum efficiency. An avalanche photodiode (APD) is used at 1064 $\mathrm{nm}$ as PMT detectors have poor quantum efficiency at that wavelength. The APD has good dynamic range and quantum efficiency but the dark noise is much larger than for the PMTs. Thus the $532 \mathrm{~nm}$ channels are more sensitive.

CALIOP is required to accurately measure signal returns from the aerosol-free region between $30 \mathrm{~km}$ and $35 \mathrm{~km}$ as well as the strongest cloud returns. For this reason, all detectors are used in analog mode, although the electronic gains of the $532 \mathrm{~nm}$ channels are large enough to allow detection of single photoelectron events. The signal processing electronics have been designed so the linear dynamic range encompasses the full range of molecular, aerosol, and cloud backscattering encountered in the atmosphere, which spans about six orders of magnitude. Dual 14-bit digitizers on each channel provide the effective 22-bit dynamic range which is required. Table II lists specifications of the receiver subsystem.

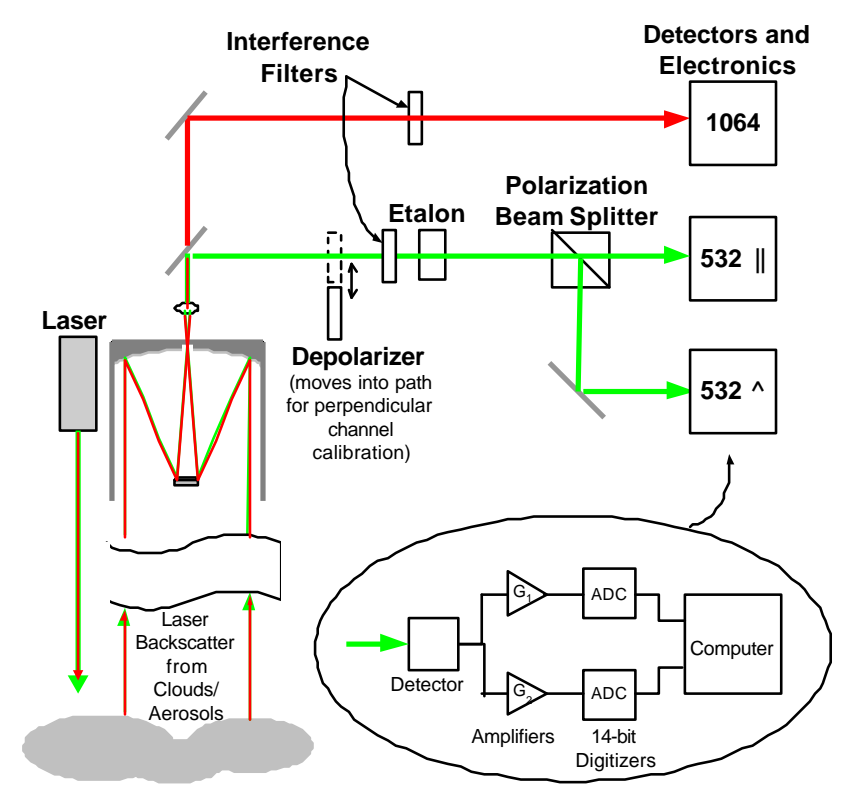

Figure 2. Functional block diagram of CALIOP.

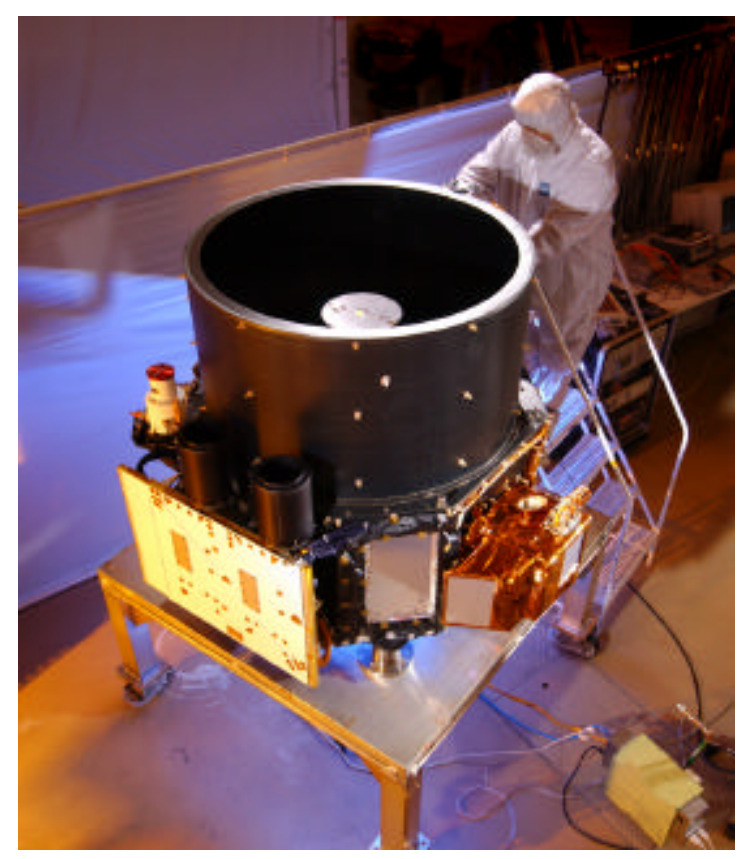

Figure 3. Completed CALIOP payload under test in BATC facilities. 
Table II. CALIOP receiver parameters

\begin{tabular}{|ll|}
\hline Telescope diameter & 1 meter \\
Field of View & $130 \mu \mathrm{rad}$ \\
Digitization Rate & $10 \mathrm{MHz}$ \\
Linear Dynamic Range & $4 \mathrm{E}+6: 1$ \\
532 nm Channel: & \\
$\quad$ Detector & PMT \\
Etalon Passband & $37 \mathrm{pm}$ \\
Etalon Peak Transmission & $85 \%$ \\
$\quad$ Blocking Filter & $770 \mathrm{pm}$ \\
1064 nm Channel: & \\
$\quad$ Detector & APD \\
Optical Passband & $450 \mathrm{pm}$ \\
Peak Transmission & $84 \%$ \\
\hline
\end{tabular}

As-built resource requirements for the laser system, for CALIOP, and for the entire payload are listed in Table III. Mass is listed for the laser system consisting of two laser heads and redundant power supply and control electronics. Payload mass includes $11 \mathrm{~kg}$ for star trackers, which are attached to the telescope sunshield. Power requirements are given at beginning of life (BOL). The laser power requirement is for one laser, since only one laser is operated at a time.

Table III. Resource requirements

\begin{tabular}{|c|c|c|c|}
\hline & Laser system & CALIOP & Payload \\
\hline Mass (kg) & 35 & 172 & 283 \\
\hline Power (W), BOL & 99 & 197 & 241 \\
\hline Dimensions (cm) & $\begin{array}{l}\text { laser footprint: } \\
20.8 \mathrm{~W} \text { x } 48.9 \mathrm{~L}\end{array}$ & $\begin{array}{c}100 \mathrm{~W} \times 149 \mathrm{~L} \\
\times 131 \mathrm{H}\end{array}$ & $\begin{array}{c}149 \mathrm{~W} \text { x } 184 \mathrm{~L} \\
\text { x } 131 \mathrm{H}\end{array}$ \\
\hline Data rate $(\mathrm{kbps})$ & N/A & 332 & 397 \\
\hline
\end{tabular}

\section{DATA ACQUISITION AND SIGNAL PROCESSING}

A number of functions are performed by the instrument to convert the analog detector signals into the profiles which are downlinked. These include: range determination, background subtraction, digitization, merging, and averaging. There are two opposing drivers on data processing: to maximize spatial resolution and dynamic range of the signal while minimizing the telemetry data volume. Several features were implemented to reduce the required telemetry bandwidth by more than an order of magnitude relative to the raw data, while impacting the information content of the data as little as possible.

Due to the oblateness of the Earth, the range from the circular orbit of the CALIPSO satellite to mean sea level (MSL, defined by the height of the geoid) varies by about $21 \mathrm{~km}$ through the orbit. The rate of change of the range to MSL is as much as 22 meters/sec. To allow on-orbit averaging of profile data while maintaining the vertical resolution of 30 meters the data acquisition timing must be adjusted to account for the changing range to the Earth's surface. The Payload Controller makes a real-time determination of the range to MSL for each laser shot using an on-board geoid model and an orbit propagator, which is updated used data from the spacecraft GPS. The data acquisition timing is then adjusted so that each profile will have the same altitude registration with respect to the geoid.

Data acquisition timing is illustrated in Figure 4 in terms of height above MSL. A timer is started when the laser fires. When the laser pulse reaches an altitude of $115 \mathrm{~km}$ above MSL, the PMT detectors are gated on and the profile signals 
from all three channels are acquired (the APD detector has no gate and is always on). The analog profiles are sampled at $10 \mathrm{MHz}$ (corresponding to $15 \mathrm{~m}$ range interval) until the elapsed time corresponds to a range of $18.5 \mathrm{~km}$ below sea level, at which point the PMTs are gated off and the digitizers stop sampling. The samples acquired between $40 \mathrm{~km}(30 \mathrm{~km}$ for the $1064 \mathrm{~nm}$ channel) and $-2 \mathrm{~km}$ are used to create the profile data which is downlinked. The portions of the profile above $60 \mathrm{~km}$ and below $-11 \mathrm{~km}$ are used to measure DC signal levels.
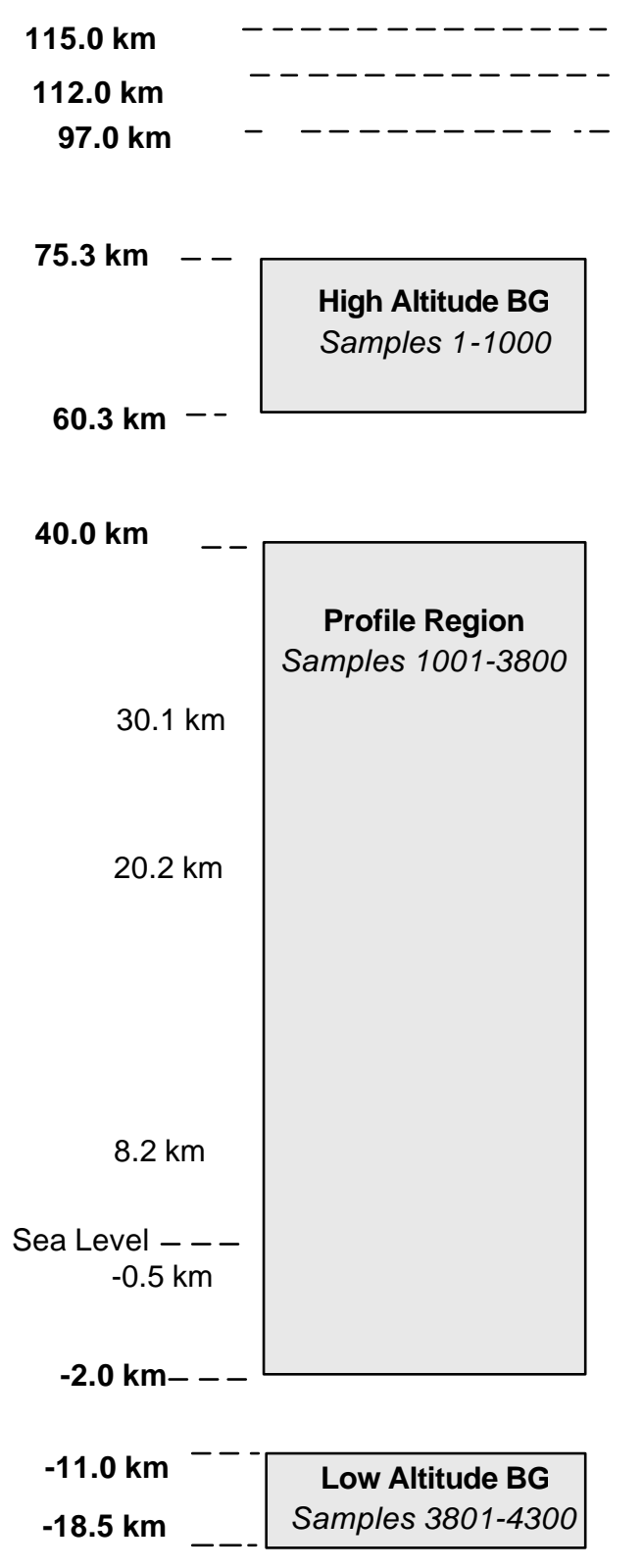

Figure 4. CALIPSO data acquisition timing. Sample numbers refer to the number of 15 -meter range bins.
The solar background signal can be significant - as large as the clear-sky atmospheric signal. The instrument measures the DC background of each profile from the signal acquired between $112 \mathrm{~km}$ and $97 \mathrm{~km}$, where the laser backscatter signals are negligible. This DC signal is electronically subtracted from the analog profile before digitization to allow the dynamic range of the digitizer to be used most effectively. This subtraction will result in negative-going noise excursions if the laser backscatter signal is small. These negative spikes will be clipped by the digitizers producing a bias of the mean signal. To avoid this, a fixed electrical offset is added, prior to digitization, to the portion of the profile below $97 \mathrm{~km}$. The magnitude of this offset is accurately measured using the 1000 samples acquired between $75.3 \mathrm{~km}$ and $60.3 \mathrm{~km}$, which are averaged and downlinked as a single value. This average of the upper background region is numerically subtracted from the profile during later processing. The 500 samples between $-11 \mathrm{~km}$ and $-18.5 \mathrm{~km}$ are also averaged and downlinked, providing another measure of the offset.

Two $10 \mathrm{MHz}$ 14-bit analog-to-digital converters (ADCs), set for different gains, are used in each channel to provide the required 22-bit effective dynamic range. On each channel, the high gain ADC measures weak signals and the low gain ADC acquires signals which saturate the high gain digitizer. The profile samples are taken from the high-gain ADC if they are on-scale. If a sample is saturated on the high-gain ADC, the corresponding sample from the low-gain ADC is used. The outputs of each pair of digitizers are re-scaled and merged into a single profile before being downlinked. The fundamental sampling resolution of the lidar is 30 meters vertical and 333 meters horizontal, determined by the receiver electrical bandwidth and the laser pulse repetition rate. Therefore, each pair of adjacent 15meter samples is averaged to produce a profile of 1400 30 -meter samples extending from $40 \mathrm{~km}$ to $-2 \mathrm{~km}$. 1064 $\mathrm{nm}$ profiles extend only from $30 \mathrm{~km}$ to $-2 \mathrm{~km}$, as 1064 $\mathrm{nm}$ returns from the purely molecular atmosphere above $30 \mathrm{~km}$ are negligibly small.

The atmosphere becomes more spatially uniform with increasing altitude. Further, signals from higher in the atmosphere tend to be weaker and require more averaging. Therefore, an altitude-dependent on-board averaging scheme was developed which provides full resolution in the lower 
troposphere - where the spatial variability of cloud and aerosol is greatest - and lower resolution higher in the atmosphere. The degree of averaging varies with altitude, as detailed in Table IV (altitudes are with respect to the geoid).

TABLE IV. Spatial resolution of downlinked data.

\begin{tabular}{|cccc|}
$\begin{array}{c}\text { Altitude } \\
\text { Range }(\mathrm{km})\end{array}$ & $\begin{array}{c}\text { Horizontal } \\
\text { Resolution }(\mathrm{km})\end{array}$ & $\begin{array}{c}532 \mathrm{~nm} \text { Vertical } \\
\text { Resolution }(\mathrm{m})\end{array}$ & $\begin{array}{c}1064 \mathrm{~nm} \text { Vertical } \\
\text { Resolution }(\mathrm{m})\end{array}$ \\
30.1 to 40.0 & 5.0 & 300 & --- \\
20.2 to 30.1 & 1.67 & 180 & 180 \\
8.2 to 20.2 & 1.0 & 60 & 60 \\
-0.5 to 8.2 & 0.33 & 30 & 60 \\
-2.0 to -0.5 & 0.33 & 300 & 300 \\
\hline
\end{tabular}

The averaging scheme provides full resolution through the depth of the planetary boundary layer and as much of the lower troposphere as possible. The lowest land elevation is the shore of the Dead Sea, with a depression of 392 meters below sea level. Therefore, the high-resolution region extends to $-0.5 \mathrm{~km}$. The region from $-0.5 \mathrm{~km}$ to $-2.0 \mathrm{~km}$ is provided at low vertical resolution to allow monitoring of potential pulse stretching from dense clouds or delayed recovery from the surface return. Data between $8.2 \mathrm{~km}$ and $20.2 \mathrm{~km}$ has a vertical resolution of 60 meters, which allows several vertical samples within even the thinnest cirrus layers. The stratosphere is much more spatially homogeneous than the troposphere. Therefore, between $20.2 \mathrm{~km}$ and $30.1 \mathrm{~km}$ the vertical resolution is degraded to 180 meters, as stratospheric aerosols and polar stratospheric clouds found in this region tend to have much greater vertical extent than the thin cirrus layers found in the upper troposphere. Only coarse spatial resolution is required above $30 \mathrm{~km}$, as the aerosol concentration is near zero. Molecular returns from this region are used for calibration of the $532 \mathrm{~nm}$ parallel channel.

\section{CALIBRATION}

CALIOP is calibrated in three steps. First, the $532 \mathrm{~nm}$ parallel channel signal is calibrated to the predicted molecular volume backscatter coefficient in the $30-34 \mathrm{~km}$ region. The molecular backscatter coefficient can be accurately estimated using temperature and pressure profiles from a gridded meteorological analysis product. The 30-34 km region was chosen as the aerosol backscatter in that region is insignificant with respect to molecular backscatter and the molecular density does not exhibit large variations. The parallel-polarized component of the molecular backscatter is derived from the estimate of total molecular backscatter by taking into account the bandwidth of the receiver optical filters. Independent estimates of the 532-nm parallel channel calibration constant are computed at approximately 700-km intervals over the dark side of each orbit and interpolated to the day side.

Second, the calibration of the 532-nm parallel channel is transferred to the perpendicular channel via insertion of a pseudo-depolarizer in the receiver optical path upstream from the polarization beamsplitter (Figure 2). The pseudodepolarizer ensures that, regardless of the polarization state of the backscatter incident on the receiver, an equal amount of light is sent to the parallel and perpendicular channels of the receiver downstream of the depolarizer. The pseudodepolarizer will be inserted periodically during the mission, to track any relative change in sensitivity of the parallel and perpendicular channel detectors.

Third, the calibration of the 532-nm channels is transferred to the 1064-nm channel via comparison of the return signals from high-altitude cirrus clouds ${ }^{2}$. Cirrus cloud particles are large compared to the transmitted wavelengths, so the backscatter coefficients will be nearly equal at $532 \mathrm{~nm}$ and $1064 \mathrm{~nm}$. By choosing clouds for which the ratio of particulate to molecular scattering is 50 and above, the calibration can be transferred with high accuracy. This calibration can be performed on both the dark and daylight side of the orbit, wherever cirrus of sufficient backscatter strength exist.

\section{ATMOSPHERIC TEST RESULTS}

Fabrication of CALIOP was completed in mid-2003, followed by an extensive series of environmental tests required for space qualification. The final step in the testing process was an end-to-end atmospheric test, performed at the BATC facilities in Boulder, Colorado during December 2003. The CALIPSO payload, pointing upward, was placed inside an environmentally controlled container with an optical-quality windows in the ceiling allowing the transmitter and receiver 
to view the sky. CALIOP was designed to operate $\sim 700 \mathrm{~km}$ outside the atmosphere, so an aperture mask was placed over the window in front of the receiver tele scope to reduce the signal from an altitude of $30 \mathrm{~km}$ to a level equivalent to that expected from orbit. The container was placed outside so that atmospheric profiles could be acquired. A number of tests were conducted over a 4 day period demonstrating the end-to-end performance of the system.

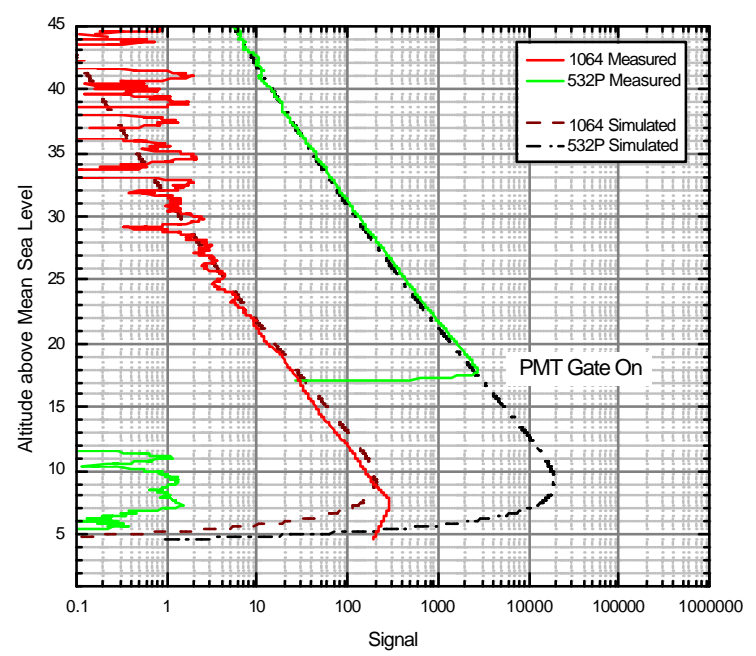

Figure 5. Clear-air atmospheric test profiles, 15-minute average, smoothed over $630 \mathrm{~m}$ vertically.

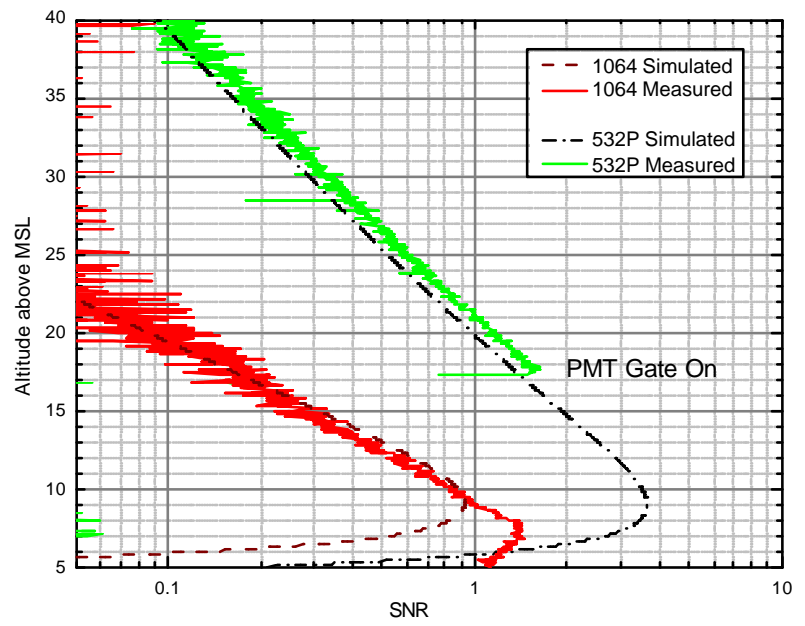

Figure 6. Comparison of measured and predicted SNR, $0.25 \mathrm{sec}$ average, no smoothing.
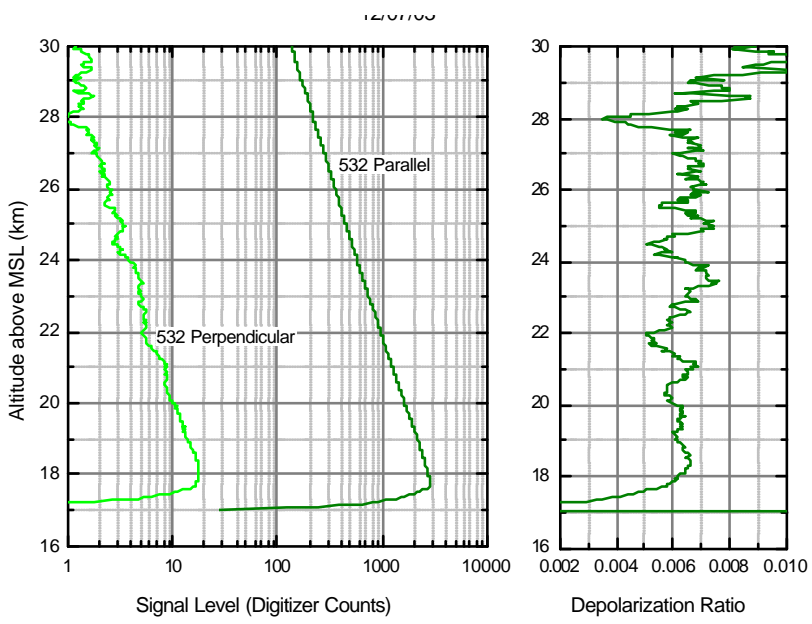

Figure 7. Left panel: Clear-air $532 \mathrm{~nm}$ parallel and perpendicular profiles acquired during atmospheric testing; Right panel: depolarization profile

Example profiles from the atmospheric testing are shown in Figures 5, 6, and 7. For atmospheric testing, the transmitter assembly was pointed slightly toward the receiver so that full overlap of the receiver field of view and the transmitted beam occurred at an altitude of about $6 \mathrm{~km}$. The $532 \mathrm{~nm}$ profiles shown in these figures were computed by summing the $532 \mathrm{~nm}$ parallel and perpendicular profiles, applying the appropriate gain ratio. The profiles shown in Fig. 5 were acquired in clear air, with the PMT detectors gated on at an altitude of about $15 \mathrm{~km}$, to minimize signal-induced noise effects from the strong low altitude returns. Dashed and dotted lines show signal profiles from an instrument 
performance model based on measured instrument component characteristics. Observed signal strength was within about $20 \%$ of signals predicted by the model, with $532 \mathrm{~nm}$ signals about $20 \%$ higher than modeled and the $1064 \mathrm{~nm}$ signal about 20\% lower. Figure 6 shows signal-to-noise ratio (SNR) computed from clear-air profiles compared with modeled SNR. Observed SNR is about 17\% larger at $532 \mathrm{~nm}$ and 11\% smaller at 1064 nm relative to modeled SNR. Fig. 7 shows clear-air parallel and perpendicular $532 \mathrm{~nm}$ profiles and the corresponding depolarization profile (defined as $\delta_{\mathrm{V}}=\beta_{\mathrm{perp}} / \beta_{\mathrm{par}}$ ). Given the spectral bandpass of the etalon use in the $532 \mathrm{~nm}$ channel we expect a molecular depolarization of about $0.39 \%$. The measured value of $0.6 \%$ indicates that indicating instrumental sources of depolarization bias are small and well within requirements.

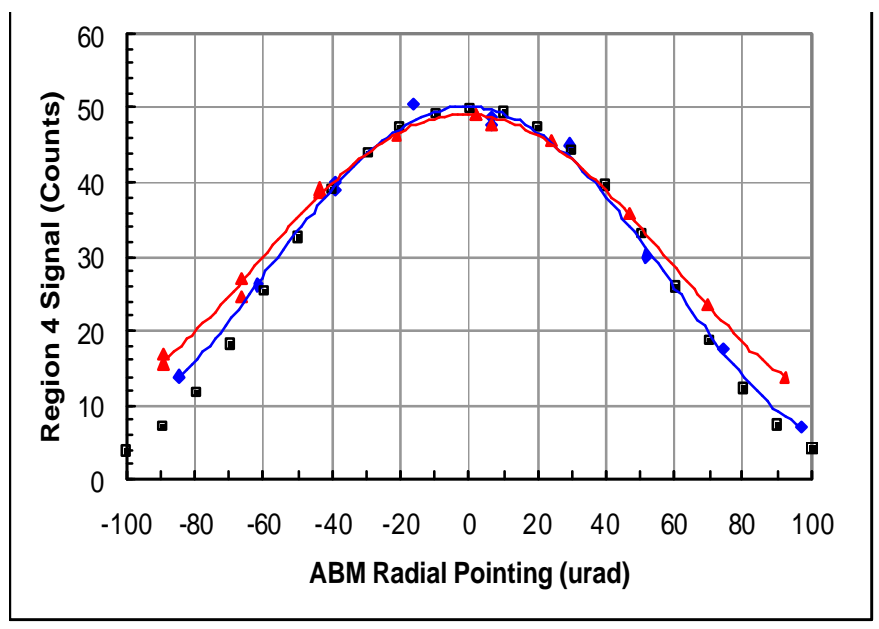

Figure 8. Beam profiles from orthogonal boresight scans (triangles, diamonds) and modeled estimate (squares) assuming Gaussian beam of $100 \mu \mathrm{rad}$ FWHM.

An important element of the atmospheric test was the operation of the boresight alignment system. An atmospheric test is the only time that operation of the boresight system can be tested as it requires receiving signals backscattered at least several kilometers from the instrument. The testing successfully demonstrated the transmitter could be accurately aligned with the receiver and that the alignment was repeatable, with an error in the final "aligned" position of less than $13 \mu \mathrm{rad}$. . The boresight system can also be used to measure the laser beam profile. Fig. 8 shows the result of a test where the beam profile was measured by scanning the transmitter across the receiver field of view in two orthogonal directions. The measured profiles show good agreement with model estimates, which assumed a $100 \mu$ rad Gaussian beam divergence.

\section{STATUS}

Integration of the CALIPSO payload and platform was accomplished in February 2004. Space qualification of the satellite was completed in September 2004. CALIPSO is planned for launch in spring 2005 from VAFB on a Delta II launch vehicle in a dual-payload configuration with the CloudSat satellite.

\section{ACKNOWLEDGEMENTS}

I would like to acknowledge the tremendous effort put into this project by the engineering teams at Ball Aerospace and Technology Corporation, at NASA Langley Research Center, and at Fibertek, whose talents and dedication were key to the success of this effort.

\section{REFERENCES}

1. Winker, D. M., J. Pelon, and M. P. McCormick, "The CALIPSO mission: Spaceborne lidar for observation of aerosols and clouds," Proc. SPIE, 4893, 1-11, 2003.

2. Reagan, J. A., X. Wang, and M. T. Osborn, "Spaceborne lidar calibration from cirrus and molecular backscatter returns," Trans. Geosci.. \& Rem. Sens., 40, 2285-2290, 2002. 